\title{
Biomechanical consideration for dorsal-lumbar and lumbar sagittal spine disorders
}

\author{
C. Bignardi ${ }^{1}$, A. Ramieri ${ }^{2}$ \& G. Costanzo ${ }^{2}$ \\ ${ }^{I}$ Department of Mechanics, Politecnico di Torino, Torino, Italy \\ ${ }^{2}$ Department of Orthopaedics, University "La Sapienza"- \\ Polo Pontino ICOT of Latina and Don Gnocchi Foundation, Rome, Italy
}

\begin{abstract}
Lower back pain is one of the most important socioeconomic diseases and one of the most important health care issues today. On average, $50-90 \%$ of the adult population suffers from lower back pain and lifetime prevalence of lower back pain is $65-80 \%$. The causes of lower back pain often remain unclear and may vary from patient to patient. It is estimated that $75 \%$ of such cases are associated with lumbar degenerative intervertebral disk disease. Dysmorphisms or sagittal disorders of the rachis mean back (kyphosis) or front (lordosis) pathological deviations, irreducible in various measures, resulting from structural diskligament and vertebral alterations of various etiologies. Three numerical models of the dorsal-lumbar spine, respectively a physiological model, a ipolordotic model and a kyphotic model, have been realized considering bone, disks and ligaments with their specific mechanical characteristics. For the load and boundary conditions chosen, joint facets and intervertebral disk stresses and disk bulge have been compared for the three spine situations. When it has been possible, the obtained results have been validated with data available in literature regarding both experimental and computational studies. In conclusion it can be assumed that dorsal-lumbar and lumbar sagittal spine disorders can determine premature disks and joints alterations. In particular, it seems that dorsal-lumbar kyphosis, more than lumbar ipolordosis, can expose joint facets and disks to non physiological loads. In addition, if the genetic role of disk degeneration is acknowledged, it is probable that the correction, at a precocious age, of sagittal spine imbalance, can prevent or slacken the disk-joint lumbar-sacral degenerative phenomena. The obtained results agree with the clinical experience.
\end{abstract}

Keywords: spine sagittal disorders, biomechanics, FEM. 


\section{Introduction}

The concept of sagittal alignment and spine balancing has been introduced in literature in order to define the limit of normality of kyphosis and spinal lordosis. Different studies have been carried out regarding the thoracic and lumbar spine, but few have been carried out concerning the cervical spine, with evaluations of asymptomatic subjects, often volunteers, during growth or as adults. Only quite recent observations have highlighted how spinal-pelvic balancing alterations are responsible for premature lumbar intervertebral disk degenerations, lumbago or spondylolisthesis $[3,4]$.

The basic loading modes acting on the spine, while performing daily activities, are axial compression, flexion/extension, lateral bending and torsion. Load is transferred from one vertebral end-plate to the next by means of nucleus pulposus and annulus fibrosus. Load produces complex stresses within the annular ring.

In order to compare joint facets and intervertebral disks stresses and disks bulging for different spine situations, three numerical models of the dorsallumbar spine, respectively a physiological model, an ipolordotic model and a kyphotic model, have been realized considering bone, disks and ligaments with their specific mechanical characteristics. The greatest difficulty in the numerical modelling of the spine is found in the simulation of the intervertebral disk.

Compression testing has been the most commonly used method for the study of mechanical behaviour of the intervertebral disk [5-8], but many experiments have been also done subjecting the intervertebral disk to bending and torsional loads and to pure share loading [9-13]. Its viscoelastic nature has also been determined [14]; typically, all viscoelastic structures exhibit hysteresis, intervertebral disks also show this phenomenon in which there is loss of energy after repetitive loading-unloading cycles. Hysteresis has been observed to vary with the applied load, age and disk level [15].

Considering the complex structure of the intervertebral disk and the diverse stresses to which it is subjected under physiological loading conditions, it is clear that experimental techniques alone are not sufficient to fully characterize the overall mechanical behaviour of the motion segment. This is corroborated by the technical complexities that precluded the measurement of the stress state, deformation and disk bulge at different locations throughout the motion segment. This provided the motivation for the development of numerical methods, such as finite element analysis, to expand the experimental data in order to characterize the intervertebral disk parameters, which may be difficult to measure experimentally. Many researchers have simulated the intervertebral disk mechanics using the finite element method. Belytschko et al [16] were the first to use the finite element method for understanding the intervertebral disk mechanics. The disk-body unit was assumed to be an axisymmetric object and annulus as a linear orthotropic material. This model was further expanded to accommodate the nonlinear (orthotropic) properties for the annulus, keeping all other parameters unchanged [17]. Different approaches were followed by Lin et al [18] and by Spilker [19]. The first attempt to make a realistic finite element model of the lumbar intervertebral disk, considering the composite nature of the 
annulus fibrosus, was made by Shirazi et al [20]. For the first time, this model accounted for both material and geometric nonlinearities along with the representation of the annulus as a composite of collagen fibres embedded in a matrix of ground substance. The nucleus was modelled as an incompressible, inviscid fluid. The model was based on the lumbar L2-L3 functional spinal unit. This model was compared to the experimental observations of load-displacement behaviour, disk bulge, end-plate bulge and intradiscal pressure. Stress and strain distribution in the cortical/cancellous bones, end-plates, annulus fibres and annulus ground substance were reported under compressive load. The same model was expanded to assess the effect of axial torque in combination with compression [21] and sagittal plane moments [22]. There were also significant efforts by other researchers to understand the intervertebral disk mechanics by taking into consideration the experimental results and physiological conditions of other spine components. Crisco and Panjabi [23] compared the lateral stabilizing potential of the lumbar spine muscles as a function of the architecture. Stabilizing effects of muscles on the overall mechanics of a lumbar spine were observed by Goel et al [24].

A viscoelastic model to study the changes in load sharing during the fast and slow loading rate was analyzed by Wang et al [25]. It has been known for a long time that the biphasic nature (solid and fluid phase) of the disk components plays a major role in the loading mechanism of the hydrated intervertebral disk. In the late 1980s, there was an increasing interest in the modelling of the disk as a saturated porous media by using the poroelastic approach [26-28]. Important models are those worked out by Eberlein et al [29], Vena et al [30], Lavaste et al [31] and Cao et al [32].

Many authors have studied pathological conditions of the functional spine unit related to disk degeneration processes, osteoporotic condition, end-plate fractures and different resections of the iatrogenic nature [33-38], but at the moment, as far as we know, the influence on joint facets and intervertebral disk stresses and the disk bulge of spinal-pelvic balancing alterations has not been studied.

\section{Materials and methods}

Starting from a CAD model of a spine available in the web [39], realized with CT images regarding a healthy 35-year-old male subject, a FEM model of the spine zone T11-S1, that later on we will call "physiological", has been worked out. The external three-dimensional geometry of vertebral bodies and disks from the starting CAD model have been utilized; spongious bone, nucleus pulposus and annulus fibrosus, constituents of the intervertebral disk, endplates, ligaments and muscles have been drawn afterwards. In order to verify the geometric reliability of the CAD model, it has been scaled and placed upon the RX and MR images that we had at our disposal, regarding a healthy subject, see fig. 1a), b).

Afterwards the model was modified, based on RX and MR images that we had at our disposal, making the spinal curve pathologic, respectively ipolordotic and kyphotic; two further FEM models have been so realized, see fig. 2. 


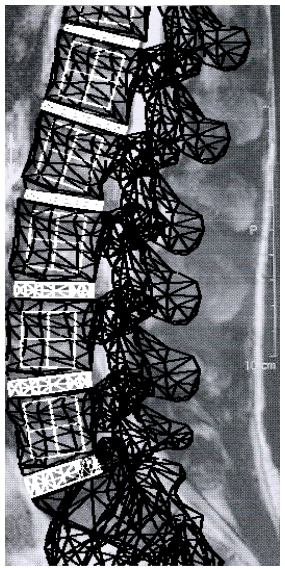

a)

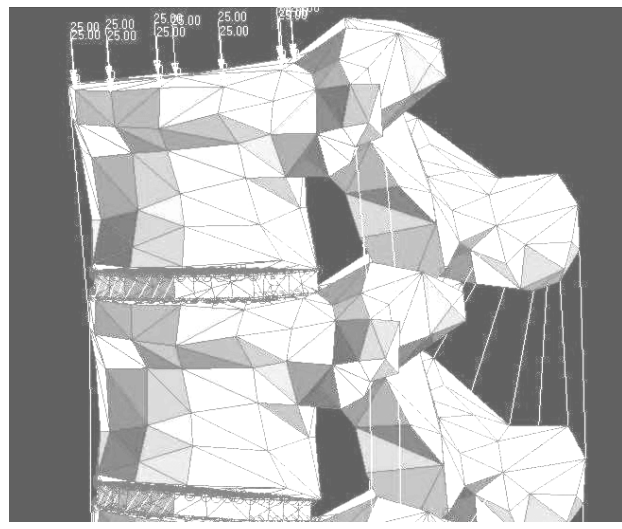

b)

Figure 1: a) Lumbar zone of the "physiological" FE model realized placed upon an MR image of a healthy subject, b) detail of T11-T12 spinal unit that shows ligament distributions and applied loads.

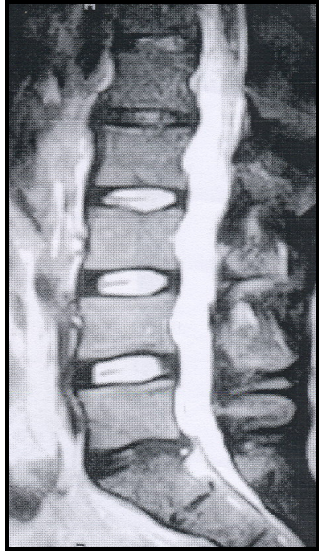

a)

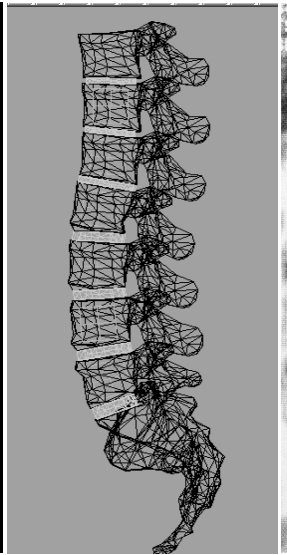

b)

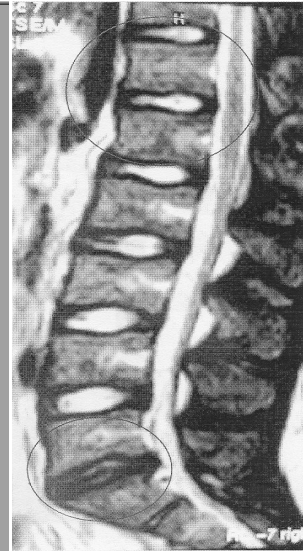

c)

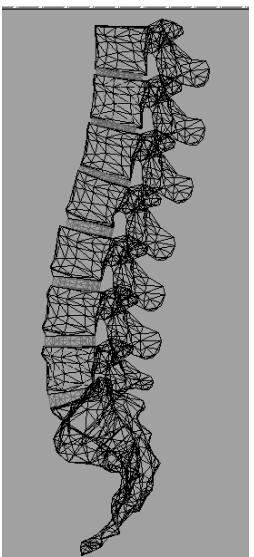

d)

Figure 2: a) RM image of an ipolordotic spine, b) FE model of an ipolordotic spine, c) RM image of a dorsal-lumbar kyphotic spine, d) FE model of a dorsal-lumbar kyphotic spine that compensates with an ipolordosis.

As regards the modelling of annulus fibrosus, the type of model suggested by Shirazi et al [20] has been adopted; three concentric layers of fibrous tissue in which bundles of connective fibres runs sideways from the nucleus to the outside, alternating their directions in the contiguous layers so as to form respectively $45^{\circ}-70^{\circ}-120^{\circ}$ angles with regard to a horizontal plane, have been simulated. 
The modelled materials are listed in table 1; mechanical properties agree with data found in the literature [20].

Ligaments and muscles considered in the modelling, fig. 1b), which give intrinsic stability to the spine, are shown in table 2 with their geometric and mechanical characteristics according with literature [23,24,40-42].

Table 1: $\quad$ Mechanical properties of materials.

\begin{tabular}{lrc}
\hline Material & $\begin{array}{c}\text { Young's modulus } \\
{[\mathrm{MPa}]}\end{array}$ & $\begin{array}{c}\text { Poisson's } \\
\text { ratio }\end{array}$ \\
\hline Cortical bone & 12000 & 0.3 \\
Spongious bone & 100 & 0.3 \\
Endplate & 23000 & 0.3 \\
Annulus matrix & 4 & 0.4 \\
Annulus fibres & 450 & 0.499 \\
Nucleus & 4 & 0.499 \\
\hline
\end{tabular}

Table 2: $\quad$ Geometric and mechanical properties of ligaments and muscles.

\begin{tabular}{lccc}
\hline \multicolumn{1}{c}{ Material } & $\begin{array}{c}\text { Cross section } \\
\text { area }\left[\mathrm{mm}^{2}\right]\end{array}$ & $\begin{array}{c}\text { Young's } \\
\text { modulus } \\
{[\mathrm{MPa}]}\end{array}$ & $\begin{array}{c}\text { Poisson's } \\
\text { ratio }\end{array}$ \\
\hline Interspinous ligaments & 36.3 & 11.6 & 0.3 \\
Intertransversal ligaments & 2 & 58.7 & 0.3 \\
Anterior longitudinal ligament & 25.5 & 20 & 0.3 \\
Posterior longitudinal ligament & 9.2 & 20 & 0.3 \\
Sovraspinosus ligament & 75.7 & 15 & 0.3 \\
Spinal muscles & 75.7 & 0.1 & 0.3 \\
\hline
\end{tabular}

Solid elements have been used for the discretization of bone, nucleus pulposus, annulus matrix and endplates, while bar elements have been used for annulus fibres, ligaments and muscles.

The three models have been bound in correspondence with the sacrum and loaded with a vertical distributed force $(350 \mathrm{~N})$ in correspondence with T11.

For the modelling, the commercial softwares Rhinoceros (Robert McNeel \& Associates, Seattle, USA) and Patran/Nastran (MSC Software Corporation, Santa Ana, USA) have been used.

\section{Results and discussion}

Joint facets and intervertebral disks stresses and disk bulge have been compared for the three spine situations.

In figure 3 the results are shown as regards joint facets, in terms of mean von Mises stress values computed in the contact areas. 


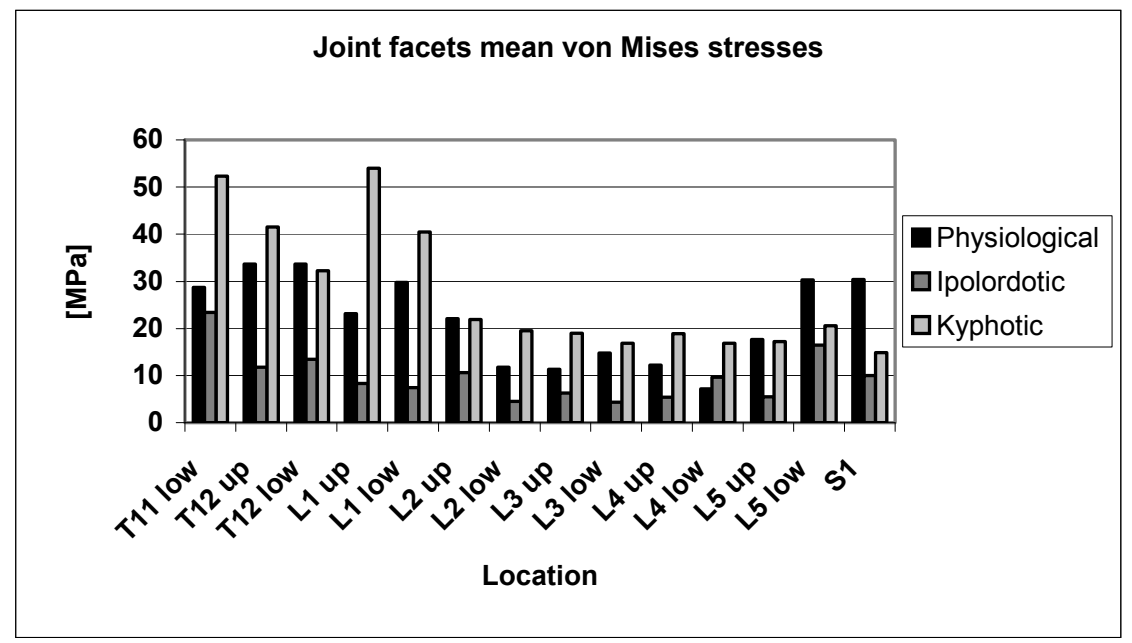

Figure 3: Comparison among mean von Mises stresses on joint facets, lower and upper, for the three sagittal spine configurations.

From the analysis of the diagram it can be observed how, in the physiological model, stress values are highest in correspondence with the thoracic zone and the sacrum and lower in correspondence with the lumbar zone. As regards the kyphotic model, generally intervertebral facets appear more stressed than the other two models, except in correspondence with the sacrum, where we find a reduction of stress state compared with the physiological model. This result could be caused by the fact that in that zone rachis compensates with an ipolordosis. As regards the ipolordotic model, stresses on facets appear in every zone of the spine lower than in the other two models. Results have been compared and validated with results obtained by Goto et al [43] as regards a physiological model of a L4-L5 functional spinal unit.

In figure 4 the results are shown as regards intervertebral disks, in terms of mean von Mises stresses; the values shown represent the average stress of the whole intervertebral disk, including nucleus pulposus and annulus fibrosus. Both pathological models show a remarkable increase of stress values in correspondence of all zones of the spine if compared with those obtained with the physiological model. For the kyphotic model in particular, it is evident that there is high stress in correspondence of L5-S1 where, what's more, the MR images at our disposal showed a disk degeneration. In this case we compared and validated our results with those obtained by Natarajan and Andersson [44] as regards a physiological model of a L3-L4 functional spinal unit.

As regards disk bulge, both pathological models show a general increase, both in the sagittal and in the frontal plane, if compared with the disk bulge obtained for the physiological model; in particular, the increase is higher in correspondence of those zones that RX and MR images suggest at the risk of disk degeneration. For example, in table 3 data are shown obtained for the L4-L5 


\section{Intervertebral disks mean von Mises stresses}

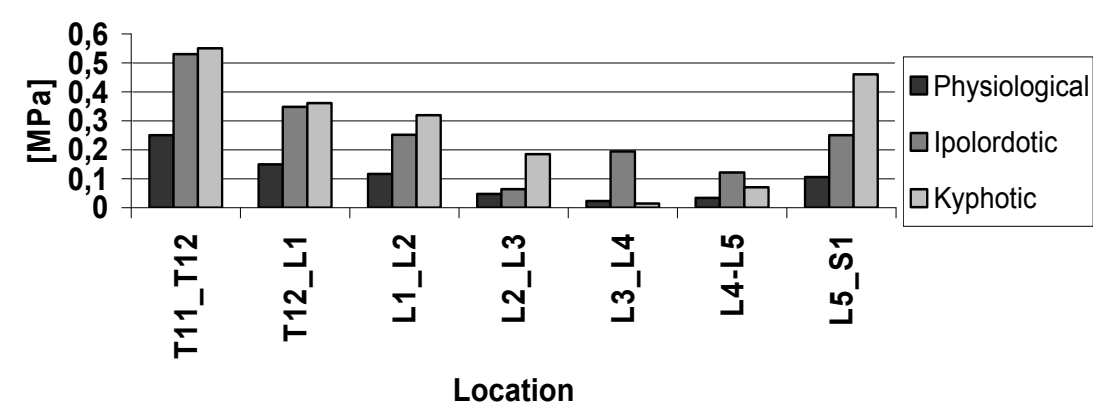

Figure 4: Comparison among intervertebral disks; mean von Mises stresses for the three sagittal spine configurations.

Table 3: Sagittal disk bulge $[\mathrm{mm}]$ for the three spine configurations in L4-L5.

\begin{tabular}{llllll}
\hline \multicolumn{2}{l}{ Physiological model } & \multicolumn{2}{l}{ Kyfotic model } & \multicolumn{2}{l}{ Ipolordotic model } \\
\hline Front & Back & Front & Back & Front & Back \\
0,99 & 0,99 & 1,32 & 1,32 & 1,32 & 2,47 \\
\hline
\end{tabular}

functional spinal unit. In this case we compared and validated our results with those obtained by Shirazi et al [21] as regards a physiological model of a L2-L3 functional spinal unit.

\section{Conclusions}

The analysis carried out proves how geometric variations of the rachis curve, both at lumbar and thoracic level, conditions a lot of the stress distribution of the intervertebral disk and of the joint facets. In particular, simulations carried out with the realized models have shown a local increase of disk stresses and bulge in those zones where RX and MR images at our disposal showed degenerated disks. In the ipolordotic model, joint facet stresses are on average lower than in the physiological model, while in the kyphotic model, stresses reach higher values in correspondence of T12-L1. Intervertebral disk stresses are on average higher in the ipolordotic and in the kyphotic models than in the physiological model. Nevertheless, stresses are particularly higher in the kyphotic model in correspondence of T12-L1 and L5-S1. As regards bulge, the highest values are in L4-L5 and L5-S1. In conclusion it can be assumed that dorsal-lumbar and lumbar sagittal spine disorders can determine premature disk and joint alterations. In particular, it seems that the dorsal-lumbar kyphosis, more than the lumbar ipolordosis, can expose joint facets and disks to non physiological loads.

In addition, if the genetic role of disk degeneration is acknowledged, it is probable that the correction, at a precocious age, of sagittal spine imbalance, can prevent or slacken the disk-joint lumbar-sacral degenerative phenomena. 


\section{References}

[1] Heliovaara, M., Makela, M., Knekt, P., Impivaara, O. \& Aromaa, A. Determinants of sciatica and low-back pain. Spine, 16(6), pp. 608-614, 1991.

[2] Manchikanti, L. Epidemiology of low back pain. Pain Physician, 3(2), pp. 167-192, 2000.

[3] Rajnics, P., Templier, A., Skalli, W., Lavaste, F. \& Illes, T. The importance of spinopelvic parameters in patients with lumbar disc lesions. International Orthopaedics, 26(2), pp. 104-108, 2002.

[4] Rajnics, P., Templier, A., Skalli, W., Lavaste, F. \& Illés, T. The association of sagittal spinal and pelvic parameters in asymptomatic persons and patients with isthmic spondylolisthesis. Journal of Spinal Disorders \& Techniques, 15(1), pp. 24-30, 2002.

[5] Brown, T., Hanson, R. \& Yorra, A. Some mechanical tests on the lumbosacral spine with particular reference to the intervertebral discs. The Journal of Bone and Joint Surgery, 39A (5), pp. 1135-1164, 1957.

[6] Farfan, H.F. Mechanical Disorders of the Low Back, Lea and Febiger, Philadelphia, 1973.

[7] Hirsch, C. The reaction of intervertebral discs to compression. The Journal of Bone and Joint Surgery, 37A (6), pp.1188-1896, 1955.

[8] Hirsch, A. \& Nachemson, A.L. New observation on the mechanical behaviour of the lumbar discs. Acta orthopaedica Scandinavica, 23, pp. 254-283, 1954.

[9] Galante, J.O. Tensile properties of the human annulus fibrosus. Acta orthopaedica Scandinavica, Suppl. 100, pp. 4-91, 1967.

[10] Fujita Y., Duncan, N.A. \& Lotz, J.C. Radial tensile properties of the lumbar annulus fibrosus are site and degeneration dependent. Journal of Orthopaedic Research, 15(6), pp. 814-819, 1997.

[11] Roaf R. A study of the mechanics of spinal injuries. The Journal of Bone and Joint Surgery, 42B, pp. 810-823, 1960.

[12] Farfan, H.F., Cossette, J.W., Robertson, G.H., Wells, R.V. \& Kraus, H. The effect of torsion on the lumbar intervertebral joints: the role of torsion in the production of disc degeneration. The Journal of Bone and Joint Surgery, 52A, pp. 468-497, 1970.

[13] White, A.A. \& Panjabi, M.M. Clinical Biomechanics of the Spine. II ed. J.B. Lippincott Company, Philadelphia, 1990.

[14] Markolf, K.L. \& Morris, J.M. The structural components of the intervertebral disc: a study of their contributions to the ability of the disc to withstand compressive forces. The Journal of Bone and Joint Surgery, 56A, pp. 675-687, 1974.

[15] Virgin, W. Experimental investigations into physical properties of intervertebral disc. The Journal of Bone and Joint Surgery, 33B(4), pp. 607-611, 1951 . 
[16] Belytschko, T.B., Kulak, R.F., Schultz, A.B. \& Galante, J.O. Finite element stress analysis of an intervertebral disc. Journal of biomechanics, 7(3), pp. 277-285, 1974.

[17] Kulak, R.F., Belytschko, T.B., Schultz, A.B. \& Galante, J.O. Nonlinear behaviour of the human intervertebral disc under axial load. Journal of biomechanics, 9(6), pp. 377-386, 1976.

[18] Lin, H.S., Liu, Y.K. \& Adams, K.H. Mechanical response of the lumbar intervertebral joint under physiological (complex) loading. The Journal of Bone and Joint Surgery, 60A, pp. 41-55, 1978.

[19] Spilker, R.L. A simplified finite element model of the intervertebral disc. In Finite Elements in Biomechanics, ed. Simon, B.R., Tuscon, University of Arizona, pp. 729-747, 1980.

[20] Shirazi, A., Shrivastava, S.C. \& Ahmed, S.M. Stress analysis of the lumbar disc-body unit in compression. Spine, 9(2), pp. 120-134, 1984.

[21] Shirazi, A., Ahmed, A.M. \& Shrivastava, S.C. Mechanical response of a lumbar motion segment in axial torque alone and combined with compression. Spine, 11(9), pp. 914-927, 1986.

[22] Shirazi, A., Ahmed, A.M. \& Shrivastava, S.C. A Finite Element study of a lumbar motion segment subjected to pure sagittal plane moments. Journal of Biomechanics, 19(4), pp. 331-350, 1986.

[23] Crisco, J.J. \& Panjabi, M.M. The intersegmental and multisegmental muscles of the lumbar spine: a biomechanical model comparing lateral stabilizing potential. Spine, 16(7), pp. 793-799, 1991.

[24] Goel, V.K., Kong, W., Han, J.S., Weinstein, J.N. \& Gilbertson, L.G. A combined Finite Element and optimization investigation of lumbar spine mechanics with and without muscles. Spine, 18(11), pp. 1531-1541, 1993.

[25] Wang, J.L., Parnianpour, M., Shirazi, A., Engin, A.E., Li, S. \& Patwardhan, A. Development and validation of a viscoelastic Finite Element model of an L2/L3 motion segment. Theoretical and Applied Fracture Mechanics, 28, pp. 81-93, 1997.

[26] Simon, B.R., Carlton, M.W., Wu, J.S.S., Evans, J.H. \& Kazarian, L.E. Structural models for human spinal motion segments based on a poroelastic view of the intervertebral disc. Journal of Biomechanical Engineering, 107, pp. 327-335, 1985.

[27] Laible, J.P., Pflaster, D.S., Krag, M.H., Simon, B.R., Pope, M.H. \& Haugh, L.D. A poroelastic swelling Finite Element model with application to the intervertebral disc. Spine, 18(5), pp. 659-670, 1993.

[28] Argoubi, M. \& Shirazi, A. Poroelastic creep response analysis of a lumbar motion segment in compression. Journal of Biomechanics, 29(10), pp. 1331-1339, 1996.

[29] Eberlein, R., Holzapfel, G.A. \& Fröhlich, M. Multi-segment FEA of the human lumbar spine including the heterogeneity of the annulus fibrosus. Computational Mechanics, 34, pp. 147-163, 2004.

[30] Vena, P., Franzoso, G., Gastaldi, D., Contro, R. \& Dallolio, V. A finite element model of the L4-L5 spinal motion segment: biomechanical 
compatibility of an interspinous device. Computer methods in biomechanics and biomedical engineering, 8(1), pp. 7-16, 2005.

[31] Lavaste, F., Skalli, W., Robin, S., Roy-Camille, R. \& Mazel, A. Threedimensional geometrical and mechanical modelling of the lumbar spine. Journal of. Biomechanics, 25(10), pp.1153-1164, 1992.

[32] Cao, K.D., Grimm, M.J. \& Yang, K. Load sharing within a human lumbar vertebral body using the Finite Element Method. Spine, 26(12), pp. 253260, 2001.

[33] Mizrahi, J., Silva, M.J., Keaveny, T.M., Edwards, W.T. \& Hayes, W.C. Finite Element stress analysis of the normal and osteoporotic lumbar intervertebral body. Spine, 18(14), pp. 2088-2096, 1993.

[34] Natarajan, R.N., Ke, J.H. \& Andersson, G.B.J. A model to study the disc degeneration process. Spine, 19(3), pp. 259-265, 1994.

[35] Goel, V.K., Monroe, B.T., Gilbertson, L.G. \& Brinckmann, P. Interlaminar shear stresses and laminae separation in a disc: Finite Element analysis of the L3-L4 motion segment subjected to axial compressive loads. Spine, 20(6), pp. 689-698, 1995.

[36] Lu, Y.M., Hutton, W.C. \& Gharpuray, V. Can variations in intervertebral disc height affect the mechanical function of the disc? Spine, 21(19), pp. 2208-2217, 1996.

[37] Natarajan, R.N. \& Andersson, G.B.J. Modelling the annular incision in a herniated lumbar intervertebral disk to study its effect on disk stability. Computers and Structures, 64(5/6), pp. 1291-1297, 1997.

[38] Pitzen, T., Geisler, F., Matthis, D., Muller-Storz, H., Barbier, D., Steudel, W. \& Feldges, A. A Finite Element model for predicting the biomechanical behaviour of the human lumbar Spine. Control Engineering Practice, 10, pp. 83-90, 2002.

[39] http://homepages.ulb.ac.be/ anatemb/exchange.htm

[40] Pintar, F.A., Yoganandan, N., Myers, T., Elhagediab, A. \& Sances, A.J. Biomechanical properties of human lumbar spine ligaments. Journal of Biomechanics, 25(11), pp.1351-1356, 1992.

[41] Brolin, K. \& Halldin, P. Development of a Finite Element Model of the upper cervical spine and parameter study of ligament characteristics. Spine, 29(4), pp. 376-385, 2004.

[42] Natarajan, S., Dargahi, J. \& Heidari B. Biomechanical effect of posterior elements and ligamentous tissues of lumbar spine on load sharing. Biomedical materials and engineering, 15(3), pp.145-158, 2005.

[43] Goto, K., Tajma, N., Chosa, E., Totoribe, K., Kuroki, H., Arizumi, Y. \& Arai, T. Mechanical analysis of the lumbar vertebrae in a three-dimensional Finite Element Method model in which intradiscal pressure in the nucleus polposus was used to establish the model, Journal of Orthopaedic Science, 7, pp. 243-246, 2002.

[44] Natarajan, R.N. \& Andersson, G.B.J. The influence of lumbar disc height and cross-sectional area on the mechanical response of the disc to physiologic loading, Spine, 24(18), pp. 1873-1881, 1999. 